\title{
TG05
}

\section{Identification of Sweet Spots over a Basin Center Gas Play Utilizing Simultaneous Seismic Inversion}

\author{
M. Al Otaibi* (Saudi Aramco), M. Al Duhailan (Saudi Aramco) \& M. Al
} Mahmoud (Saudi Aramco)

\section{SUMMARY}

Simultaneous inversion of 3D seismic data was utilized to identify sweet spots within a Permo -

Carboniferous clastic reservoir which is identified as a potential basin-centered gas play in Saudi Arabia.

Both acoustic and shear impedances resulting from simultaneous inversion were used to generate seismic sections depicting the product of Lame' parameters (mu,lambda)and density(rho).

Sweet spots of porous hydrocarbon bearing clastic reservoirs can be identified in the lambda*rho and mu* rho domains as having relatively low lambda*rho and $\mathrm{mu}^{*}$ rho. These inversion results were calibrated with rock physics modeling work. 


\section{EAGE}

Simultaneous inversion of 3D seismic data was utilized to identify sweet spots within a Permo Carboniferous clastic reservoir which is identified as a potential basin-centered gas play in Saudi Arabia. Both acoustic and shear impedances resulting from simultaneous inversion were used to generate seismic sections depicting the product of Lamé parameters $(\lambda, \mu)$ and density $(\rho)$.

A rock physics feasibility study including fluid substitution modeling and cross-plot analysis was carried out over eight wells, prior to conducting seismic inversion. A mud invasion correction was performed to correct the log readings to account for the mud invaded environment of in-situ reservoir conditions. Shear logs were predicted using different transforms and fluid substitution from brine to gas was carried out using Gassmann's equation over all eight wells. Modeling results showed that $\lambda \rho$ was the most sensitive elastic modulus when substituting gas for brine. Results also revealed that $\mu \rho$ was highly sensitive to porosity variation but not to the pore fluid type. Sweet spots of porous hydrocarbon-bearing clastic reservoirs can therefore be identified as having relatively low $\lambda \rho$ and $\mu \rho$. Based on the eight wells, a transform was generated to relate porosity to $\mu \rho$ and another to compute water saturation from $\lambda \rho$.

More than 30 wells and several angle stacks were utilized to run simultaneous seismic inversion for the study area. Inverted seismic sections of $\lambda \rho$ and $\mu \rho$ have shown excellent correlation with porosity and water saturation logs. Transforms generated from the rock physics analysis were used to create porosity and water saturation volumes from the $\mu \rho$ and $\lambda \rho$ estimates, respectively. 